\title{
UNIFORM CUSP PROPERTY, BOUNDARY INTEGRAL, AND COMPACTNESS FOR SHAPE OPTIMIZATION
}

\author{
Michel C. Delfour* \\ Centre de recherches mathématiques et Département de mathématiques et de statistique \\ Université de Montréal, C.P. 6128, succ. Centre-ville, Montréal (Qc), Canada H3C 3J7 \\ delfour@CRM.UMontreal.CA
}

Nicolas Doyon

Département de mathématiques et de statistique, Université de Montréal, C. P. 6128, succ. Centre-ville, Montréal (Qc), Canada H3C $3 J^{7} 7$

nicodoyon@hotmail.com

Jean-Paul Zolésio

CNRS and INRIA, 2004 route des lucioles, BP 93, FR-06902 Sophia Antipolis, France Jean-Paul.Zolesio@sophia.inria.fr

Abstract In this paper we consider the family of sets verifying the uniform cusp
property introduced in [2] and extended in [4] to cusp functions only
continuous at the origin. In the latter case we show that to any ex-
tended cusp function, we can associate a continuous, non-negative, and
monotone strictly increasing cusp function of the type introduced in [2].
We construct an example of a bounded set in $\mathrm{R}^{\mathrm{N}}$ with a cusp function
of the form $c|\theta|^{\alpha}, 0<\alpha<1$, for which its boundary integral is infinite
and the Hausdorff dimension of its boundary is exactly $N-\alpha$. We then
give compactness theorems for the family of subsets of a bounded open
holdall verifying a uniform cusp property with a uniform bound on ei-
ther the De Georgi [6] or the $\gamma$-density perimeter of Bucur and Zolésio
[1]. We also give their uniform local $C^{0}$-graph versions following [4].

\footnotetext{
*This research has been supported by National Sciences and Engineering Research Council of Canada discovery grant $\mathrm{A}-8730$ and by a FQRNT grant from the Ministère de l'Éducation du Québec.
} 
This class forms a much larger family than the one of subsets verifying a uniform cone property.

Keywords: Oriented distance function, signed distance function, compactness, cusp property, boundary integral, density perimeter, Hausdorff dimension

\section{Introduction}

In this paper we consider the family of sets verifying the uniform cusp property introduced in [2] and extended in [4] to cusp functions only continuous at the origin. In the latter case we show that to any extended cusp function, we can associate a continuous, non-negative, and monotone strictly increasing cusp function of the type originally introduced in [2]. Unlike sets verifying a uniform cone property, such sets do not necessarily have a locally finite boundary integral. This fact is illustrated by constructing an example of a bounded subset of $\mathbf{R}^{\mathrm{N}}$ with cusp function $c|\theta|^{\alpha}, 0<\alpha<1$, for which the boundary integral is infinite and the Hausdorff dimension of its boundary is exactly $N-\alpha$.

Even without a uniform bound on the perimeter a general compactness theorem was given in [4] for a family of subsets of a bounded hold-all verifying a uniform cusp property with a cusp function only continuous at the origin. In this paper we give compactness theorems for the family of subsets of a bounded open holdall verifying a uniform cusp property with a uniform bound on either the De Georgi [6] or the $\gamma$-density perimeter of Bucur and Zolésio [1]. We also give in $\S 4.3$ their uniform local $C^{0}$-graph versions following [4]. This class of subsets forms a much larger family than the one of subsets verifying a uniform cone property.

\section{Preliminaries: Topologies on Families of Sets}

We first introduce some notation. Given an integer $N \geq 1, \mathrm{~m}_{N}$ and $H_{N-1}$ will denote the $N$-dimensional Lebesgue and $(N-1)$-dimensional Hausdorff measures. The inner product and the norm in $\mathbf{R}^{\mathrm{N}}$ will be written $x \cdot y$ and $|x|$. The complement $\left\{x \in \mathbf{R}^{\mathrm{N}}: x \notin \Omega\right\}$ and the boundary $\bar{\Omega} \cap \bar{C} \Omega$ of a subset $\Omega$ of $\mathbf{R}^{N}$ will be respectively denoted by $C \Omega$ or $\mathbf{R}^{\mathrm{N}} \backslash \Omega$ and by $\partial \Omega$ or $\Gamma$. The distance function $d_{A}(x)$ from a point $x$ to a subset $A \neq \varnothing$ of $\mathbf{R}^{\mathrm{N}}$ is defined as $\inf \{|y-x|: y \in A\}$.

Recall a few results on metric topologies defined on spaces of equivalence classes of sets constructed from the characteristic function, the distance or the oriented distance functions to a set. Given $\Omega \subset \mathbf{R}^{\mathrm{N}}$, $\Gamma \neq \varnothing$, the oriented distance function is defined as

$$
b_{\Omega}(x) \stackrel{\text { def }}{=} d_{\Omega}(x)-d_{\complement \Omega}(x)
$$


It is Lipschitz continuous of constant 1 , and $\nabla b_{\Omega}$ exists and $\left|\nabla b_{\Omega}\right| \leq 1$ almost everywhere in $\mathbf{R}^{\mathrm{N}}$. Thus $b_{\Omega} \in W_{\text {loc }}^{1, p}\left(\mathbf{R}^{\mathrm{N}}\right)$ for all $p, 1 \leq p \leq \infty$. Recall that $b_{\Omega}^{+}=d_{\Omega}, b_{\Omega}^{-}=d_{\complement \Omega}$, and $\left|b_{\Omega}\right|=d_{\Gamma}$, and that $\chi_{\text {int } \Omega}=\left|\nabla d_{\complement \Omega}\right|$, $\chi_{\text {int } C \Omega}=\left|\nabla d_{\Omega}\right|$, and $\chi_{\Gamma}=1-\left|\nabla d_{\Gamma}\right|$ a.e. in $\mathbf{R}^{\mathrm{N}}$, where $\chi_{A}$ denotes the characteristic function of a subset $A$ of $\mathbf{R}^{\mathrm{N}}$. Given a nonempty subset $D$ of $\mathbf{R}^{\mathrm{N}}$, the family $C_{b}(D)=\left\{b_{\Omega}: \Omega \subset \bar{D}\right.$ and $\left.\Gamma \neq \varnothing\right\}$ is closed in $W^{1, p}(D)$. The following theorem is central. It shows that convergence and compactness in the metric on $C_{b}(D)$ associated with $W^{1, p}(D)$ will imply the same properties in the other topologies introduced in [2].

THEOREM 1 Let $D \subset \mathbf{R}^{\mathrm{N}}$ be bounded open and $1 \leq p<\infty$. The maps

$$
\begin{gathered}
b_{\Omega} \mapsto\left(b_{\Omega}^{+}, b_{\Omega}^{-},\left|b_{\Omega}\right|\right)=\left(d_{\Omega}, d_{\complement \Omega}, d_{\partial \Omega}\right): C_{b}(D) \subset W^{1, p}(D) \rightarrow W^{1, p}(D)^{3} \\
b_{\Omega} \mapsto\left(\chi_{\partial \Omega}, \chi_{\operatorname{int} \Omega}, \chi_{\operatorname{int} \complement \Omega}\right): W^{1, p}(D) \rightarrow L^{p}(D)^{3}
\end{gathered}
$$

are continuous.

Proof. - They are well-defined from [2] (Chapter 5, Theorem 2.1 (iii), p. 207) for the map (2) and [2] (Chapter 5, Thm 2.2 (iv)-(v), p. 210) for the map (3). They are continuous from [2] (Chapter 5, Thm 5.1).

\section{Extension of the Uniform Cusp Property}

The uniform cusp property introduced in [2] (Chapter 5, § 11) was specified by a continuous function $h:[0, \rho[\rightarrow \mathbf{R}$ such that

$$
h(0)=0, \quad h(\rho)=\lambda, \quad \forall \theta, 0<\theta<\rho, \quad 0<h(\theta)<\lambda .
$$

Recall that with $h$ of the form $h(\theta)=\lambda(\theta / \rho)^{\alpha}, 0<\alpha \leq 1$, we recover the uniform cusp property for $0<\alpha<1$ and the uniform cone property for $\alpha=1, \rho=\lambda \tan \omega$ and $h(\theta)=\theta / \tan \omega$ which corresponds to an open cone in 0 of aperture $\omega$, height $\lambda$, and axis $e_{N}$.

The uniform cusp property was extended in [4] to the family of cusp functions $h$ in the larger space

$$
\mathcal{H} \stackrel{\text { def }}{=}\{h:[0, \infty[\rightarrow \mathbf{R}: h(0)=0 \text { and } h \text { is continuous in } 0\}
$$

by associating with $h \in \mathcal{H}, \rho>0$, and $\lambda$ the axi-symmetrical region

$$
C(\lambda, h, \rho) \stackrel{\text { def }}{=}\left\{\left(\zeta^{\prime}, \zeta_{N}\right) \in \mathbf{R}^{\mathrm{N}}:\left|\zeta^{\prime}\right|<\rho \text { and } \limsup _{\xi^{\prime} \rightarrow \zeta^{\prime}} h\left(\left|\xi^{\prime}\right|\right)<\zeta_{N}<\lambda\right\}
$$

around the axis $e_{N}=(0, \ldots, 0,1)$ in $\mathbf{R}^{\mathrm{N}}$. Given $\lambda>0, \rho>0, h \in \mathcal{H}$, and a direction $d \in \mathbf{R}^{\mathrm{N}},|d|=1$, the rotated region from direction $e_{N}$ to 
$d$ is defined as

$$
C(\lambda, h, \rho, d) \stackrel{\text { def }}{=}\left\{y \in \mathbf{R}^{\mathrm{N}}: \begin{array}{l}
\left|P_{H_{d}}(y)\right|<\rho \text { and } \\
\limsup _{z \rightarrow y} h\left(\left|P_{H_{d}}(z)\right|\right)<y \cdot d<\lambda
\end{array}\right\},
$$

where $H_{d}=\{d\}^{\perp}$ is the hyperplane through 0 orthogonal to the direction $d$. Finally, the translation of $C(\lambda, h, \rho, d)$ to the point $x$ will be denoted

$$
C_{x}(\lambda, h, \rho, d) \stackrel{\text { def }}{=} x+C(\lambda, h, \rho, d) .
$$

Lemma 2 ([4],[5]) For all $\lambda>0, \rho>0, h \in \mathcal{H}$, and $x \in \mathbf{R}^{\mathrm{N}}$, the regions $C(\lambda, h, \rho)$ and $C_{x}(\lambda, h, \rho, d)$ are nonempty and open. Moreover the segment $(x, x+\lambda d)$ is contained in $C_{x}(\lambda, h, \rho, d)$.

The function $h$ is referred to as a cusp function and the space $\mathcal{H}$ as the space of cusp functions. The definition of the uniform cusp property in [2] (Chapter $5, \S 11$ ) can now be extended to the larger class $\mathcal{H}$.

Definition 3 Let $\Omega$ be a subset of $\mathbf{R}^{\mathrm{N}}$ such that $\partial \Omega \neq \varnothing$.

(i) $\Omega$ satisfies the local uniform cusp property if

$\forall x \in \partial \Omega, \quad \exists h \in \mathcal{H}, \exists \lambda>0, \exists \rho>0, \exists r>0, \exists d \in \mathbf{R}^{\mathrm{N}},|d|=1$, such that $\forall y \in B(x, r) \cap \bar{\Omega}, \quad C_{y}(\lambda, h, \rho, d) \subset \operatorname{int} \Omega$.

(ii) Given $h \in \mathcal{H}, \Omega$ satisfies the $h$-local uniform cusp property if $\forall x \in \partial \Omega, \quad \exists \lambda>0, \exists \rho>0, \exists r>0, \exists d \in \mathbf{R}^{\mathrm{N}},|d|=1$, such that $\forall y \in B(x, r) \cap \bar{\Omega}, \quad C_{y}(\lambda, h, \rho, d) \subset \operatorname{int} \Omega$.

(iii) $\Omega$ satisfies the uniform cusp property for $(r, \lambda, h, \rho)$ if

$$
\begin{gathered}
\exists h \in \mathcal{H}, \exists \lambda>0, \exists \rho>0, \exists r>0, \quad \forall x \in \partial \Omega, \exists d \in \mathbf{R}^{\mathrm{N}},|d|=1, \\
\text { such that } \quad \forall y \in B(x, r) \cap \bar{\Omega}, \quad C_{y}(\lambda, h, \rho, d) \subset \text { int } \Omega .
\end{gathered}
$$

The three cases of Definition 3 only differ when $\partial \Omega$ is not compact.

THEOREM 4 ([4]) If $\partial \Omega$ is compact, then the three uniform cusp properties of Definition 3 coincide.

In fact, when a local uniform cusp property is verified for some cusp function $h \in \mathcal{H}$, it is verified for another cusp function which is continuous, non-negative, and monotone strictly increasing as in (4).

ThEOREM 5 Assume that $\Omega$ satisfies the local uniform cusp property in $x \in \partial \Omega$ for some $(r, \lambda, h, \rho), h \in \mathcal{H}$. Then there exist $\left(r^{\prime}, \lambda^{\prime}, h^{\prime}, \rho^{\prime}\right)$, 
with $h^{\prime} \in \mathcal{H}$ continuous, non-negative, monotone strictly increasing, and $\lambda^{\prime}=h^{\prime}\left(\rho^{\prime}\right)$, such that $\Omega$ satisfies the local uniform cusp property in $x \in \partial \Omega$ for $\left(r, \lambda^{\prime}, h^{\prime}, \rho^{\prime}\right)$.

Proof. - By continuity of $h \in \mathcal{H}$ in 0 ,

$$
\begin{gathered}
\exists 0<\theta_{0} \leq \rho, \quad \forall 0 \leq \theta \leq \theta_{0}, \quad|h(\theta)| \leq \lambda / 2, \\
\forall n \geq 1, \quad \exists 0<\theta_{n}<\theta_{n-1} / 2, \quad \forall 0 \leq \theta \leq \theta_{n}, \quad|h(\theta)| \leq \lambda / 2^{n+1} .
\end{gathered}
$$

At each step $n \geq 0$ construct the continuous monotone strictly increasing and non-negative function $k_{n}:\left[0, \theta_{0}\right] \rightarrow \mathbf{R}$ defined as follows

$$
k_{n}(\theta) \stackrel{\text { def }}{=} \begin{cases}\frac{\lambda}{2^{j+1}} \frac{\theta_{j}-\theta}{\theta_{j}-\theta_{j+1}}+\frac{\lambda}{2^{j}} \frac{\theta-\theta_{j+1}}{\theta_{j}-\theta_{j+1}}, & \text { if } \theta_{j+1}<\theta \leq \theta_{j}, \quad 0 \leq j \leq n-1 \\ \frac{\lambda}{2^{n+1}} \frac{\theta_{n}-\theta}{\theta_{n}}+\frac{\lambda}{2^{n}} \frac{\theta}{\theta_{n}}, & \text { if } 0 \leq \theta \leq \theta_{n} .\end{cases}
$$

By continuity of $h$ at the origin and the fact that $h(0)=0, \theta_{n} \rightarrow 0$ and $k_{n}(0) \rightarrow 0$. By construction, $0 \leq|h(\theta)| \leq k_{n+1}(\theta) \leq k_{n}(\theta)$ in $\left[0, \theta_{0}\right], k_{n+1}(\theta)=k_{n}(\theta)$ in $\left[\theta_{n+1}, \theta_{0}\right]$, and $\left\|k_{n+1}-k_{n}\right\|_{C\left[0, \theta_{n+1}\right]} \leq \lambda / 2^{n+1}$. Therefore there exists a continuous non-negative and monotone strictly increasing function $k \in C\left[0, \theta_{0}\right]$ such that $k_{n} \rightarrow k$ in $C\left[0, \theta_{0}\right], k(0)=0$, and $|h(\theta)| \leq k(\theta) \leq \lambda$ in $\left[0, \theta_{0}\right]$. Finally, if $k\left(\theta_{0}\right)=\lambda$, choose $\rho^{\prime}$ such that $k\left(\rho^{\prime}\right)=\lambda, \lambda^{\prime}=\lambda$, and $h^{\prime}=k$. If $k\left(\theta_{0}\right)<\lambda$, choose $\rho^{\prime}=\theta_{0}, \lambda^{\prime}=k\left(\theta_{0}\right)$, and $h^{\prime}=k$. From the construction, $\rho^{\prime} \leq \rho, \lambda^{\prime} \leq \lambda, h^{\prime} \geq h$, and hence $C\left(\lambda^{\prime}, h^{\prime}, \rho^{\prime}\right) \subset C(\lambda, h, \rho)$. Therefore the local uniform cusp property of Definition 3 is verified with a non-negative, continuous, and monotone strictly increasing cusp function of the form (4).

We now turn to the compactness theorem. Given a bounded open subset $D$ of $\mathbf{R}^{\mathrm{N}}, \rho>0, \lambda>0, r>0$, and $h \in \mathcal{H}$, consider the family

$$
L(D, \lambda, h, \rho, r) \stackrel{\text { def }}{=}\left\{\Omega \subset \bar{D}: \begin{array}{c}
\Omega \text { satisfies the uniform cusp } \\
\text { property for }(\lambda, h, \rho, r)
\end{array}\right\} .
$$

The compactness Theorem 11.1 ([2], Chapter 5) readily extends to $\mathcal{H}$.

THEOREM 6 ([4]) Let $D$ be a nonempty bounded open subset of $\mathbf{R}^{\mathrm{N}}$ and $1 \leq p<\infty$. For $\rho>0, \lambda>0$, and $h \in \mathcal{H}$ the family

$$
B(D, \lambda, h, \rho, r) \stackrel{\text { def }}{=}\left\{b_{\Omega}: \forall \Omega \in L(D, \lambda, h, \rho, r)\right\}
$$

is compact in $C(\bar{D})$ and $W^{1, p}(D)$. As a consequence the families

$$
\begin{aligned}
& B_{d}(D, \lambda, h, \rho, r) \stackrel{\text { def }}{=}\left\{d_{\Omega}: \forall \Omega \in L(D, \lambda, h, \rho, r)\right\}, \\
& B_{d}^{c}(D, \lambda, h, \rho, r) \stackrel{\text { def }}{=}\left\{d_{C \Omega}: \forall \Omega \in L(D, \lambda, h, \rho, r)\right\}, \\
& B_{d}^{\partial}(D, \lambda, h, \rho, r) \stackrel{\text { def }}{=}\left\{d_{\partial \Omega}: \forall \Omega \in L(D, \lambda, h, \rho, r)\right\}
\end{aligned}
$$


are compact in $C(\bar{D})$ and $W^{1, p}(D)$, and the following families are compact in $L^{p}(D)$

$$
\begin{aligned}
X(D, \lambda, h, \rho, r) & \stackrel{\text { def }}{=}\left\{\chi_{\Omega}: \forall \Omega \in L(D, \lambda, h, \rho, r)\right\}, \\
X^{c}(D, \lambda, h, \rho, r) & \stackrel{\text { def }}{=}\left\{\chi_{\complement \Omega}: \forall \Omega \in L(D, \lambda, h, \rho, r)\right\} .
\end{aligned}
$$

\section{Extended Uniform Cusp Property and Boundary Integral (Perimeter)}

Domains $\Omega$ which are locally Lipschitzian epigraphs or, equivalently, satisfy the local uniform cone property enjoy the additional property that the $(N-1)$-Hausdorff measure of their boundary $\partial \Omega$ is locally finite. In general, this is no longer true for domains which are locally Hölderian epigraphs of exponent $\alpha, 0<\alpha<1$, but we have an upper bound on the Hausdorff dimension of $\partial \Omega$. We first recall a definition.

Definition 7 Let $\Omega \subset \mathbf{R}^{\mathrm{N}}$ be such that $\partial \Omega \neq \varnothing$. The set $\Omega$ is said to be locally a $C^{0, \ell}$-epigraph, $0 \leq \ell \leq 1$, if for each $x \in \partial \Omega$ there exist

(a) an open neighborhood $\mathcal{U}(x)$ of $x$;

(b) a direction $e_{N}(x) \in \mathbf{R}^{\mathrm{N}},\left|e_{N}(x)\right|=1$;

(c) a bounded open neighborhood $V_{H(x)}$ of 0 in the hyperplane $H(x)=\left\{e_{N}(x)\right\}^{\perp}$ through 0 such that

$$
\mathcal{U}(x) \subset\left\{y \in \mathbf{R}^{\mathrm{N}}: P_{H(x)}(y-x) \in V_{H(x)}\right\},
$$

where $P_{H(x)}$ is the orthogonal projection onto $H(x)$; and

(d) a $C^{0, \ell}$-mapping $a_{x}: V_{H(x)} \rightarrow \mathbf{R}$ such that

$$
\begin{array}{r}
\mathcal{U}(x) \cap \partial \Omega=\left\{x+\zeta^{\prime}+\zeta_{N} e_{N}(x): \begin{array}{c}
\zeta^{\prime} \in V_{H(x)} \\
\zeta_{N}=a_{x}\left(\zeta^{\prime}\right)
\end{array}\right\} \\
\mathcal{U}(x) \cap \operatorname{int} \Omega=\mathcal{U}(x) \cap\left\{x+\zeta^{\prime}+\zeta_{N} e_{N}(x): \begin{array}{c}
\zeta^{\prime} \in V_{H(x)} \\
\zeta_{N}>a_{x}\left(\zeta^{\prime}\right)
\end{array}\right\} .
\end{array}
$$

THEOREM 8 If $\Omega$ in $\mathbf{R}^{\mathrm{N}}$ satisfies the uniform cusp property associated with the function $h(\theta)=\theta^{\alpha}, 0<\alpha<1$, then the Hausdorff dimension of $\partial \Omega$ is less or equal to $N-\alpha$.

Proof. - From Theorem 3.3 (i) in [4], $\Omega$ is locally a $C^{0, \alpha}$-epigraph and, a fortiori, a $C^{0}$-epigraph. Let $r>0, \rho>0$, and $\lambda>0$ be the parameters, 
$e_{N}(x)=d_{x}$ the direction and $H(x)$ the hyperplane through 0 orthogonal to $d_{x}$ associated with the point $x \in \partial \Omega$. Then there exists $\bar{\rho}$,

$$
0<\bar{\rho} \leq r_{\lambda} \stackrel{\text { def }}{=} \min \{r, \lambda / 2\}
$$

which is the largest radius such that

$$
B_{H(x)}(0, \bar{\rho}) \subset\left\{P_{H(x)}(y-x): \forall y \in B\left(x, r_{\lambda}\right) \cap \partial \Omega\right\} .
$$

The neighborhoods of Definition 3.2 in [4] or Definition 5.2 in Chapter 2 of [2] that specify the local graph $a_{x}: V_{H(x)} \rightarrow \mathbf{R}$ can be chosen as

$$
\begin{gathered}
V_{H(x)} \stackrel{\text { def }}{=} B_{H(x)}(0, \bar{\rho}) \text { and } \\
\mathcal{U}(x) \stackrel{\text { def }}{=} B\left(x, r_{\lambda}\right) \cap\left\{y: P_{H(x)}(y-x) \in V_{H(x)}\right\},
\end{gathered}
$$

where $B_{H(x)}(0, \bar{\rho})$ is the open ball of radius $\bar{\rho}$ in the hyperplane $H(x)$. For each $\zeta^{\prime} \in V_{H(x)}$, there exists a unique $y_{\zeta^{\prime}} \in \partial \Omega \cap \mathcal{U}(x)$ such that $P_{H(x)}\left(y_{\zeta^{\prime}}-x\right)=\zeta^{\prime}$ and the function

$$
\zeta^{\prime} \mapsto a_{x}\left(\zeta^{\prime}\right) \stackrel{\text { def }}{=}\left(y_{\zeta^{\prime}}-x\right) \cdot d_{x}: V_{H(x)} \rightarrow \mathbf{R}
$$

is well-defined, bounded,

$$
\forall \zeta^{\prime} \in V_{H(x)}, \quad\left|a_{x}\left(\zeta^{\prime}\right)\right|<r_{\lambda},
$$

uniformly continuous in $V_{H(x)}$, and

$$
\forall \zeta_{1}^{\prime}, \zeta_{2}^{\prime} \in V_{H(x)}, \quad\left|a_{x}\left(\zeta_{2}^{\prime}\right)-a_{x}\left(\zeta_{1}^{\prime}\right)\right| \leq c\left|\zeta_{2}^{\prime}-\zeta_{1}^{\prime}\right|^{\alpha} .
$$

Since $\partial \Omega$ is compact there exists a finite number of points $\left\{x_{i} \in \partial \Omega\right.$ : $1 \leq i \leq m\}$ such that $\partial \Omega \subset \cup_{i=1}^{m} \mathcal{U}\left(x_{i}\right)$. Given $\varepsilon<\bar{\rho}, \bar{\rho}$ as chosen in (12), let $N_{\Omega}(\varepsilon)$ be the number of hypercubes of dimension $N$ and side $\varepsilon$ required to cover $\partial \Omega$ and let $N_{\Omega, i}(\varepsilon)$ be the number of hypercubes of dimension $N$ and side $\varepsilon$ required to cover $\partial \Omega \cap \mathcal{U}\left(x_{i}\right)$.

We have the following estimate

$$
N_{\Omega, i}(\varepsilon) \leq\left(\frac{r_{\lambda}}{\varepsilon}\right)^{N-1} \frac{c(\sqrt{N-1} \varepsilon)^{\alpha}}{\varepsilon} .
$$

Indeed the neighborhood

$$
V_{H(x)}=B_{H(x)}(0, \bar{\rho}) \subset B_{H(x)}\left(0, r_{\lambda}\right)
$$

can be covered by $\left[r_{\lambda} / \varepsilon\right]^{N-1}(N-1)$-dimensional hypercubes of side $\varepsilon$. On each $(N-1)$-dimensional hypercube of side $\varepsilon$ the variation between the minimum and the maximum of the function $a_{x}$ is bounded by

$$
c\left(\sqrt{(N-1) \varepsilon^{2}}\right)^{\alpha}=c(\sqrt{N-1} \varepsilon)^{\alpha} .
$$


So the number of $N$-dimensional hypercubes of side $\varepsilon$ necessary to cover the hypersurface above each $(N-1)$-dimensional hypercube of side $\varepsilon$ is

$$
\left[\frac{c}{\varepsilon}(\sqrt{N-1} \varepsilon)^{\alpha}\right]
$$

Finally

$$
\begin{aligned}
N_{\Omega, i}(\varepsilon) & \leq\left(\frac{r_{\lambda}}{\varepsilon}+1\right)^{N-1}\left(\frac{c}{\varepsilon}(\sqrt{N-1} \varepsilon)^{\alpha}+1\right) \\
& \leq \frac{1}{\varepsilon^{N-1}} \frac{1}{\varepsilon^{1-\alpha}}\left(r_{\lambda}+\varepsilon\right)^{N-1}\left(c(\sqrt{N-1})^{\alpha}+\varepsilon^{1-\alpha}\right) \\
& \leq \frac{1}{\varepsilon^{N-\alpha}}\left(r_{\lambda}+\varepsilon\right)^{N-1}\left(c(\sqrt{N-1})^{\alpha}+\varepsilon^{1-\alpha}\right) .
\end{aligned}
$$

As a result for all $\beta>N-\alpha$

$$
\begin{aligned}
N_{\Omega, i}(\varepsilon) \leq & \sum_{i=1}^{m} N_{\Omega, i}(\varepsilon) \\
\leq & m \frac{1}{\varepsilon^{N-\alpha}}\left(r_{\lambda}+\varepsilon\right)^{N-1}\left(c(\sqrt{N-1})^{\alpha}+\varepsilon^{1-\alpha}\right) \\
\Rightarrow N_{\Omega}(\varepsilon) \varepsilon^{\beta} \leq & \varepsilon^{\beta-N+\alpha} m\left(r_{\lambda}+\varepsilon\right)^{N-1}\left(c(\sqrt{N-1})^{\alpha}+\varepsilon^{1-\alpha}\right) \\
& \Rightarrow \forall \beta>N-\alpha, \quad H_{\beta}(\partial \Omega)=0 .
\end{aligned}
$$

This means that, by definition, the Hausdorff dimension of $\partial \Omega$ is less or equal to $N-\alpha$.

It is possible to construct examples of sets verifying the uniform cusp property for which the Hausdorff dimension of the boundary is strictly greater than $N-1$ and hence $H_{N-1}(\partial \Omega)=+\infty$.

EXAMPLE 9 This following two-dimensional example of an open domain satisfying the uniform cusp condition for the function $h(\theta)=\theta^{\alpha}, 0<$ $\alpha<1$, can easily be generalized to an $N$-dimensional example. Consider the open domain $\Omega$ in $\mathbf{R}^{2}$

$$
\begin{aligned}
\Omega \stackrel{\text { def }}{=} & \{(x, y):-1<x \leq 0 \text { and } 0<y<2\} \\
& \cap\{(x, y): 0<x<1 \text { and } f(x)<y<2\} \\
& \cap\{(x, y): 1 \leq x<2 \text { and } 0<y<2\}
\end{aligned}
$$

where $f:[0,1] \rightarrow \mathbf{R}$ is defined as follows

$$
f(x) \stackrel{\text { def }}{=} d_{C}(x)^{\alpha}, \quad 0 \leq x \leq 1,
$$

and $C$ is the Cantor set on the interval $[0,1]$. This function is equal to 0 


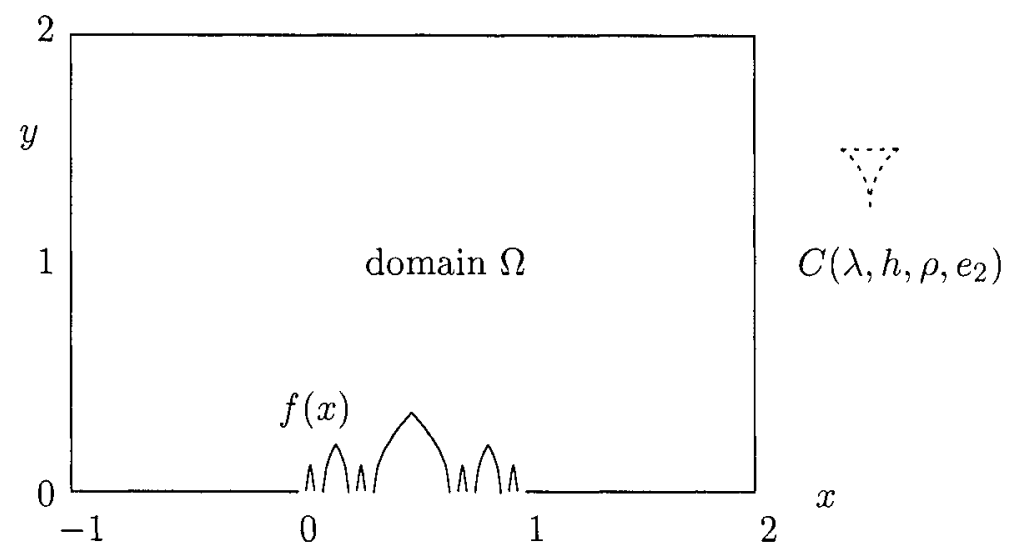

Figure 1. Domain $\Omega$ for $N=2,0<\alpha<1, e_{2}=(0,1), \rho=1 / 6, \lambda=(1 / 6)^{\alpha}$, $h(\theta)=\theta^{\alpha}$.

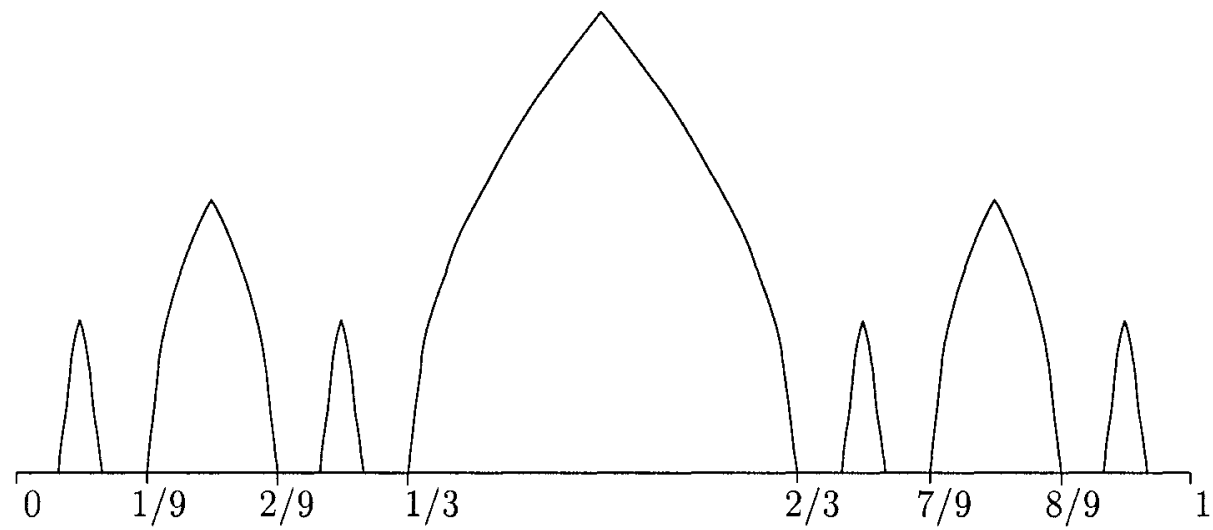

Figure 2. $f(x)=d_{C}(x)^{1 / 2}$ constructed on the Cantor set $C$ for $2 k+1=3$.

on $C$. Any point in $[0,1] \backslash C$ belongs to one of the intervals of length $3^{-k}$, $k \geq 1$, which has been deleted from $[0,1]$ in the sequential construction of the Cantor set. Therefore the distance function $d_{C}(x)$ is equal to the distance function to the two end points of that interval. In view of this special structure it can be shown that

$$
\forall x, y \in[0,1], \quad\left|d_{C}(y)^{\alpha}-d_{C}(x)^{\alpha}\right| \leq|y-x|^{\alpha}
$$

Denote by $\Gamma$ the piece of the boundary $\partial \Omega$ specified by the function $f=$ $d_{C}$. On $\Gamma$ the uniform cusp condition is verified with $\rho=1 / 6, \lambda=$ $(1 / 6)^{\alpha}$, and $h(\theta)=\theta^{\alpha}$. Clearly the number $N_{\Omega}(\varepsilon)$ of hypercubes of 
dimension $N$ and side $\varepsilon$ required to cover $\partial \Omega$ is greater than the number $N_{\Gamma}(\varepsilon)$ of hypercubes of dimension $N$ and side $\varepsilon$ required to cover $\Gamma$. The construction of the Cantor set is done by sequentially deleting intervals. At step $k=0$ the interval $(1 / 3,2 / 3)$ of width $3^{-1}$ is removed. At step $k$ a total of $2^{k}$ intervals of width $3^{-(k+1)}$ are removed. Thus if we pick $\varepsilon=3^{-(k+1)}$ the interval $[0,1]$ can be covered with exactly $3^{(k+1)}$ intervals. Here we are interested in finding a lower bound to the total number of of squares of side $\varepsilon$ necessary to cover $\Gamma$. For this purpose we only keep the $2^{k}$ intervals removed at step $k$. Vertically it takes

$$
\left[\frac{\left(2^{-1} 3^{-(k+1)}\right)^{\alpha}}{3^{-(k+1)}}\right] \geq \frac{\left(2^{-1} 3^{-(k+1)}\right)^{\alpha}}{3^{-(k+1)}}-1
$$

Then we have for $\beta \geq 0$

$$
\begin{aligned}
N_{\Omega}(\varepsilon) \geq N_{\Gamma}(\varepsilon) & \geq 2^{k}\left(\frac{\left(2^{-1} 3^{-(k+1)}\right)^{\alpha}}{3^{-(k+1)}}-1\right) \\
& \geq 2^{k-\alpha} 3^{(k+1)(1-\alpha)}-2^{k}=\left(3^{(1-\alpha)} 2\right)^{k} 2^{-\alpha} 3^{(1-\alpha)}-2^{k} \\
\Rightarrow N_{\Omega}(\varepsilon)\left(3^{-k}\right)^{1+\beta} & \geq 3^{-k(1+\beta)}\left(\left(3^{(1-\alpha)} 2\right)^{k} 2^{-\alpha} 3^{(1-\alpha)}-2^{k}\right) \\
& \geq\left(3^{-(\alpha+\beta)} 2\right)^{k} 2^{-\alpha} 3^{(1-\alpha)}-\left(\frac{2}{3^{(1+\beta)}}\right)^{k}
\end{aligned}
$$

The second term goes to zero as $k$ goes to infinity. The first term goes to infinity as $k$ goes to infinity if $3^{-(\alpha+\beta)} 2>1$, that is, $0<\alpha+\beta<$ $\ln 2 / \ln 3$. Under this condition, $H_{1+\beta}(\partial \Omega)=H_{1+\beta}(\Gamma)=+\infty$ for all $0<\alpha<\ln 2 / \ln 3$ and all $0 \leq \beta<\ln 2 / \ln 3-\alpha$. Therefore given $0<\alpha<\ln 2 / \ln 3$

$$
\forall \beta, 0 \leq \beta+\alpha<\ln 2 / \ln 3, \quad H_{1+\beta}(\partial \Omega)=+\infty
$$

and the Hausdorff dimension of $\partial \Omega$ is strictly greater than 1 .

Given $0<\alpha<1$, it is possible to construct an optimal example of a set verifying the uniform cusp property for which the Hausdorff dimension of the boundary is exactly $N-\alpha$ and hence $H_{N-1}(\partial \Omega)=+\infty$.

EXAMPLE 10 Optimal example of a set that verifies the uniform cusp property with $h(\theta)=|\theta|^{\alpha}, 0<\alpha<1$, and whose boundary has Hausdorff dimension exactly equal to $N-\alpha$.

For that purpose, we need a generalization of the Cantor set. Denote by $C_{1}$ the Cantor set. Recall that each $x, 0 \leq x \leq 1$, can be written 
uniquely (if we make a certain convention) as

$$
x=\sum_{j=1}^{\infty} \frac{a_{j}(3, x)}{3^{j}}
$$

where $a_{j}(3, x)$ can be regarded as the jth digit of $x$ written in basis 3. From this define the Cantor set is characterized as follows

$$
x \in C_{1} \Longleftrightarrow \forall j, a_{j}(3, x) \neq 1 \text {. }
$$

Similarly for an arbitrary integer $k \geq 1$, each $x \in[0,1]$ can be uniquely written in the form

$$
x=\sum_{j=1}^{\infty} \frac{a_{j}(2 k+1, x)}{(2 k+1)^{j}}
$$

and we can define the set $C_{k}$ as

$$
x \in C_{k} \Longleftrightarrow \forall j, a_{j}(2 k+1, x) \neq k
$$

In a certain sense, if $k_{1}>k_{2}, C_{k_{1}}$ contains more points than $C_{k_{2}}$. We now use these sets to construct the family of set $D_{k}$ as follows

$$
x \in D_{1} \Longleftrightarrow 2 x \in C_{1}
$$

and for $k>1$

$$
x \in D_{k} \Longleftrightarrow 2^{k+1}\left(x-2^{k}\right) \in C_{k} .
$$

Note that, if $k_{1} \neq k_{2}, D_{k_{1}} \cap D_{k_{2}}=\varnothing$ since the $D_{k}$ 's only contain points from the interval $\left[1-2^{k-1}, 1-2^{k}\right]$. Consider now the following set

$$
D \stackrel{\text { def }}{=} \bigcup_{k=1}^{\infty} D_{k}
$$

and go back to Example 9 with the function $f$ is replaced by the function

$$
f(x) \stackrel{\text { def }}{=} d_{D}(x)^{\alpha} \text {. }
$$

Again it can be shown that

$$
\forall x, y \in[0,1], \quad\left|d_{D}(y)^{\alpha}-d_{D}(x)^{\alpha}\right| \leq|y-x|^{\alpha}
$$

Note that on the interval $\left[1-2^{k-1}, 1-2^{k}\right]$ we have $d_{D}(x)^{\alpha}=d_{D_{k}}(x)^{\alpha}$.

Denote by $\Gamma$ the piece of boundary $\partial \Omega$ specified by the function $f=d_{D}$ and $\Gamma_{k}$ the part of boundary $\partial \Omega$ specified by the function $f=d_{D}=d_{D_{k}}$ on the interval $\left[1-2^{k-1}, 1-2^{k}\right]$. Once again on $\Gamma$ the uniform cusp property is verified with $\rho=1 / 6, \lambda=(1 / 6)^{\alpha}$, and $h(\theta)=\theta^{\alpha}$. 
Clearly the number $N_{\Omega}(\varepsilon)$ of hypercubes of dimension $N$ and side $\varepsilon$ required to cover $\partial \Omega$ is greater than the number $N_{\Gamma_{k}}(\varepsilon)$ of hypercubes of dimension $N$ and side $\varepsilon$ required to cover $\Gamma_{k}$. The construction of the set $C_{k}$ is also done sequentially by deleting intervals. At step $j=0$ the interval $] k /(2 k+1),(k+1) /(2 k+1)$ [ of width $(2 k+1)^{-1}$ is removed. At step $j$ a total of $2^{j}$ intervals of width $(2 j+1)^{-(j+1)}$ are removed. If we consider the intervals that remain at step $j$, a total of $2^{j+1}$ nonempty disjoint intervals of width $\left(\frac{k}{2 k+1}\right)^{j+1}$ remain in the set $C_{k}$. Each of these intervals contains a gap of length $\left(\frac{k}{2 k+1}\right)^{j+1} \frac{1}{2 k+1}$ created at step $j+1$.

If we construct the set $D_{k}$ in the same way, at step $j$ a total of $2^{j}$ nonempty disjoint intervals of width $\left(\frac{k}{2 k+1}\right)^{j+1} \frac{1}{2^{k}}$ remain in the set $D_{k}$. Each of these intervals contains a gap of length $\left(\frac{k}{2 k+1}\right)^{j+1} \frac{1}{2^{k}(2 k+1)}$. Pick

$$
\varepsilon=\frac{1}{2^{k}}\left(\frac{k}{2 k+1}\right)^{j+1}
$$

and look for a lower bound on the number of squares of side $\varepsilon$ necessary to cover $\Gamma_{k}$. For this purpose, only consider the $2^{j+1}$ nonempty disjoint intervals remaining at step $j$. As they each contain a gap of length

$$
\left(\frac{k}{2 k+1}\right)^{j+1} \frac{1}{2^{k}(2 k+1)}
$$

vertically it takes

$$
\begin{aligned}
& {\left[\left(\left(\frac{k}{2 k+1}\right)^{j+1} \frac{1}{2^{k+1}(2 k+1)}\right)^{\alpha} 2^{k}\left(\frac{2 k+1}{k}\right)^{j+1}\right]} \\
& \geq\left(\left(\frac{k}{2 k+1}\right)^{j+1} \frac{1}{2^{k+1}(2 k+1)}\right)^{\alpha} 2^{k}\left(\frac{2 k+1}{k}\right)^{j+1}-1
\end{aligned}
$$

$\varepsilon$-cubes. Then we have for $\beta \geq 0$

$$
\begin{aligned}
N_{\Omega}(\varepsilon) & \geq N_{\Gamma}(\varepsilon) \\
& \geq 2^{j+1}\left(\left(\left(\frac{k}{2 k+1}\right)^{j+1} \frac{1}{2^{k+1}(2 k+1)}\right)^{\alpha} 2^{k}\left(\frac{2 k+1}{k}\right)^{j+1}-1\right) \\
& \geq\left(\frac{2(2 k+1) k^{\alpha}}{k(2 k+1)^{\alpha}}\right)^{j+1}\left(\frac{2^{k}}{2^{\alpha(k+1)}(2 k+1)^{\alpha}}\right)-2^{j+1} \\
& =\left(2(2 k+1)^{1-\alpha} k^{\alpha-1}\right)^{j+1}\left(\frac{2^{k(1-\alpha)-\alpha}}{(2 k+1)^{\alpha}}\right)-2^{j+1}
\end{aligned}
$$


and hence

$\varepsilon^{1+\beta} N_{\Omega}(\varepsilon)$

$\geq\left(\frac{1}{2^{k}}\left(\frac{k}{2 k+1}\right)^{j+1}\right)^{1+\beta}\left(\left(2(2 k+1)^{1-\alpha} k^{\alpha-1}\right)^{j+1}\left(\frac{2^{k(1-\alpha)-\alpha}}{(2 k+1)^{\alpha}}\right)-2^{j+1}\right)$

$\geq\left(\left(\frac{k}{2 k+1}\right)^{\alpha+\beta} 2\right)^{j+1} \frac{2^{k(1-\alpha)-\alpha}}{2^{k(1+\beta)}(2 k+1)^{\alpha}}-2^{j+1}\left(\frac{1}{2^{k}}\left(\frac{k}{2 k+1}\right)^{j+1}\right)^{1+\beta}$

The second term goes to zero as $j$ goes to infinity. The first term goes to infinity as $j$ goes to infinity if $\left(\frac{k}{2 k+1}\right)^{\alpha+\beta} 2>1$ for any integer $k$, that is, if

$$
0<\alpha+\beta<\frac{\log 2}{\log ((2 k+1) / k)} .
$$

As $k$ can be chosen arbitrarily large, the former inequality reduces to $0<\alpha+\beta<1$. Under this condition there exists an integer $k$ for which $H_{1+\beta}(\partial \Omega)=H_{1+\beta}\left(\Gamma_{k}\right)=+\infty$ for all $0<\alpha<1$ and all $0 \leq \beta<1-\alpha$. Therefore, given $0<\alpha<1 \forall \beta, 0 \leq \beta<1-\alpha, H_{1+\beta}(\partial \Omega)=+\infty$.. This implies that the Hausdorff dimension of $\partial \Omega$ is greater than or equal to $2-\alpha$ which is the upper bound we obtained in Theorem 8.

\section{Compactness under the Uniform Cusp Property and a Bound on the Perimeter}

\subsection{De Giorgi Perimeter of Caccioppoli Sets}

One of the classical notions of perimeter is the one introduced in the context of the problem of minimal surfaces for Caccioppoli sets.

Definition 11 Let $\Omega$ be a measurable subset of $\mathbf{R}^{\mathrm{N}}$. Given an open set $D$ in $\mathbf{R}^{\mathrm{N}}, \Omega$ is said to have finite perimeter with respect to $D$ if $\chi_{\Omega} \in$ $B V(D)$. This perimeter denoted by $P_{D}(\Omega)$ is given by the expression

$$
P_{D}(\Omega) \stackrel{\text { def }}{=}\left\|\nabla \chi_{\Omega}\right\|_{M^{1}(D)^{N}}
$$

where $B V(D)$ is the space of functions of total bounded variation and $M^{1}(D)$ is the space of bounded measures on $D$.

Given a bounded open subset $D$ of $\mathbf{R}^{\mathrm{N}}, \rho>0, \lambda>0, r>0, c>0$, and $h \in \mathcal{H}$, consider the family

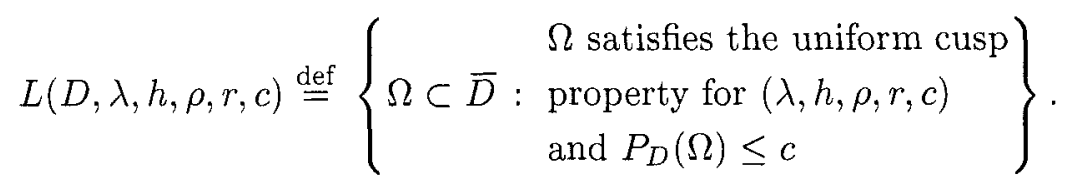


The compactness Theorem 6 readily extends to this new family.

THEOREM 12 Let $D$ be a nonempty bounded open subset of $\mathbf{R}^{\mathrm{N}}$ and $1 \leq p<\infty$. For $\rho>0, \lambda>0, c>0$, and $h \in \mathcal{H}$ and assume that $L(D, \lambda, h, \rho, r, c)$ is not empty. Then the family

$$
B(D, \lambda, h, \rho, r, c) \stackrel{\text { def }}{=}\left\{b_{\Omega}: \forall \Omega \in L(D, \lambda, h, \rho, r, c)\right\}
$$

is compact in $C(\bar{D})$ and $W^{1, p}(D)$. As a consequence the families

$$
\begin{aligned}
& B_{d}(D, \lambda, h, \rho, r, c) \stackrel{\text { def }}{=}\left\{d_{\Omega}: \forall \Omega \in L(D, \lambda, h, \rho, r, c)\right\}, \\
& B_{d}^{c}(D, \lambda, h, \rho, r, c) \stackrel{\text { def }}{=}\left\{d_{C \Omega}: \forall \Omega \in L(D, \lambda, h, \rho, r, c)\right\}, \\
& B_{d}^{\partial}(D, \lambda, h, \rho, r, c) \stackrel{\text { def }}{=}\left\{d_{\partial \Omega}: \forall \Omega \in L(D, \lambda, h, \rho, r, c)\right\}
\end{aligned}
$$

are compact in $C(\bar{D})$ and $W^{1, p}(D)$, and the following families are compact in $L^{p}(D)$

$$
\begin{aligned}
X(D, \lambda, h, \rho, r, c) & \stackrel{\text { def }}{=}\left\{\chi_{\Omega}: \forall \Omega \in L(D, \lambda, h, \rho, r, c)\right\}, \\
X^{c}(D, \lambda, h, \rho, r, c) & \stackrel{\text { def }}{=}\left\{\chi_{\complement \Omega}: \forall \Omega \in L(D, \lambda, h, \rho, r, c)\right\} .
\end{aligned}
$$

Proof. - From Theorem 6 there exist $\Omega$ in $L(D, \lambda, h, \rho, r)$ and a sequence $\left\{\Omega_{n}\right\}$ in $L(D, \lambda, h, \rho, r, c)$ such that $b_{\Omega_{n}} \rightarrow b_{\Omega}$ in $W^{1, p}(D)$ and $P_{D}\left(\Omega_{n}\right) \leq$ c. In particular, from Theorem $1, \chi_{\Omega_{n}} \rightarrow \chi_{\Omega}$ in $L^{1}(D)$. But, in view of the uniform bound $P_{D}\left(\Omega_{n}\right) \leq c$ on the $\Omega_{n}$ 's (cf. [6]), there exist a subsequence $\left\{\chi_{\Omega_{n_{k}}}\right\}$ such that $\chi_{\Omega_{n_{k}}} \rightarrow \chi_{\Omega^{\prime}}$ in $L^{1}(D)$ for some $\Omega^{\prime}$ for which $P_{D}\left(\Omega^{\prime}\right) \leq c$. But, as a subsequence of $\left\{\Omega_{n}\right\}$,

$$
b_{\Omega_{n_{k}}} \rightarrow b_{\Omega} \text { in } W^{1, p}(D) \text { and } \chi_{\Omega_{n_{k}}} \rightarrow \chi_{\Omega} \text { in } L^{1}(D) .
$$

Hence $\chi_{\Omega^{\prime}}=\chi_{\Omega}, P_{D}(\Omega)=P_{D}\left(\Omega^{\prime}\right) \leq c$, and $\Omega \in L(D, \lambda, h, \rho, r, c)$. This concludes the proof.

\subsection{Finite $\gamma$-density Perimeter}

The $\gamma$-density perimeter introduced by Bucur and Zolésio [1] is a relaxation of the $(N-1)$-dimensional upper Minkowski content which leads to the compactness Theorem 14 . We recall the definition and quote the compactness for the $W^{1, p}$-topology under a uniform bound on the $\gamma$-density perimeter as revisited in [3].

Definition 13 Let $\gamma>0$ be a fixed real and $\Omega$ a subset of $\mathbf{R}^{\mathrm{N}}$ with nonempty boundary $\Gamma$. Consider the quotient

$$
P_{\gamma}(\Gamma) \stackrel{\text { def }}{=} \sup _{0<k<\gamma} \frac{\mathrm{m}_{N}\left(U_{k}(\Gamma)\right)}{2 k} .
$$


Whenever $P_{\gamma}(\Gamma)$ is finite, we say that $\Omega$ has a finite $\gamma$-density perimeter. It was shown in [1] that, when $P_{\gamma}(\Gamma)$ is finite, $\mathrm{m}_{N}(\Gamma)=0$. The compactness result of [1] can be revisited and established in the $W^{1, p}$-topology from which convergence in all other topologies of Theorem 1 follows.

THEOREM 14 ([3]) Let $D \neq \varnothing$ be a bounded open subset of $\mathbf{R}^{\mathrm{N}}$ and $\left\{\Omega_{n}\right\}, \Gamma_{n} \neq \varnothing$, be a sequence of subsets of $\bar{D}$. Assume that

$$
\exists \gamma>0 \text { and } c>0 \text { such that } \forall n, \quad P_{\gamma}\left(\Gamma_{n}\right) \leq c .
$$

Then there exist a subsequence $\left\{\Omega_{n_{k}}\right\}$ and $\Omega, \Gamma \neq \varnothing$, of $\bar{D}$ such that

$$
\begin{gathered}
P_{\gamma}(\Gamma) \leq \liminf _{n \rightarrow \infty} P_{\gamma}\left(\Gamma_{n}\right) \leq c \\
\forall p, 1 \leq p<\infty, \quad b_{\Omega_{n_{k}}} \rightarrow b_{\Omega} \text { in } W^{1, p}\left(U_{\gamma}(D)\right) \text {-strong. }
\end{gathered}
$$

The proof of the next result combines Theorem 6 which says that the family $L(D, \lambda, h, \rho, r)$ is compact with Theorem 14 which says that the family of sets verifying (19) is compact in $W^{1, p}(D)$. The intersection of the two families of oriented distance functions is compact in $W^{1, p}(D)$.

THEOREM 15 For fixed $\gamma>0$, Theorem 12 remains true when $P_{D}(\Omega)$ is replaced by the $\gamma$-density perimeter $P_{\gamma}(\Gamma)$.

\subsection{Compactness via Local $C^{0}$-graphs}

It was shown in [4] (Thm 3.3 and 3.4) that the uniform cusp property is equivalent to conditions on the local $C^{0}$-graphs. Thus by adding a condition either on the De Giorgi or the perimeter $\gamma$-density perimeter in Theorem 4.1 of [4] we get the analogues of the above Theorems 12 and 15. Recall the definition of the orthogonal subgroup of $N \times N$ matrices

$$
\mathrm{O}(\mathrm{N}) \stackrel{\text { def }}{=}\left\{A:{ }^{*} A A=A^{*} A=I\right\},
$$

where ${ }^{*} A$ is the transposed matrix of $A$. A direction can be specified either by a matrix (of rotation) $A \in \mathrm{O}(\mathrm{N})$ or the corresponding unit vector $d=A e_{N} \in \mathbf{R}^{\mathrm{N}}$.

THEOREM 16 Let $\rho>0$ be given and assume that $U$ is a bounded neighborhood of 0 such that

$$
U \subset\left\{y \in \mathbf{R}^{\mathrm{N}}: P_{H}(y) \in B_{H}(0, \rho)\right\}, \quad V \stackrel{\text { def }}{=} B_{H}(0, \rho) .
$$

Let $R>0$ be such that $B(0,2 R) \subset U$. Given a bounded nonempty subset $D$ of $\mathbf{R}^{\mathrm{N}}$, consider a family $L(D, \rho, U)$ of subsets $\Omega$ of $\bar{D}$ with 
the following properties: for each $\Omega \in L(D, \rho, U)$ and each $x \in \partial \Omega$, there exist $A^{\Omega}(x) \in \mathrm{O}(\mathrm{N})$ and a $C^{0}$-mapping $a_{x}^{\Omega}: V^{\Omega}(x) \rightarrow \mathbf{R}$, where $V^{\Omega}(x) \stackrel{\text { def }}{=} A^{\Omega}(x) V$ and $\mathcal{U}^{\Omega}(x) \stackrel{\text { def }}{=} x+A^{\Omega}(x) U$, such that

$$
\begin{array}{r}
\mathcal{U}^{\Omega}(x) \cap \partial \Omega=\left\{x+\zeta^{\prime}+\zeta_{N} e_{N}^{\Omega}(x): \begin{array}{c}
\zeta^{\prime} \in V^{\Omega}(x) \\
\zeta_{N}=a_{x}^{\Omega}\left(\zeta^{\prime}\right)
\end{array}\right\} \\
\mathcal{U}^{\Omega}(x) \cap \operatorname{int} \Omega=\mathcal{U}^{\Omega}(x) \cap\left\{x+\zeta^{\prime}+\zeta_{N} e_{N}^{\Omega}(x): \begin{array}{c}
\zeta^{\prime} \in V^{\Omega}(x) \\
\zeta_{N}>a_{x}^{\Omega}\left(\zeta^{\prime}\right)
\end{array}\right\}
\end{array}
$$

where $e_{N}^{\Omega}(x)=A^{\Omega}(x) e_{N}$.

(i) Assume that there exists $h \in \mathcal{H}$ and $c>0$ such that

$$
\forall \Omega \in L(D, \rho, U), \forall y \in V, \quad \bar{a}_{x}^{\Omega}(y) \leq h(|y|), \quad P_{D}(\Omega) \leq c
$$

where $\bar{a}_{x}^{\Omega}=a_{x}^{\Omega} \circ A^{\Omega}(x): V \rightarrow \mathbf{R}$. Each $\Omega$ of $L(D, \rho, U)$ satisfies the uniform cusp property for the parameters $\left(r^{\Omega}, \lambda^{\Omega}, \rho^{\Omega}, h^{\Omega}\right)=$ $(R, R, \rho, h)$. Hence (from Theorem 12) the family

$$
B(D, \rho, U, c) \stackrel{\text { def }}{=}\left\{b_{\Omega}: \forall \Omega \in L(D, \rho, U) \text { and } P_{D}(\Omega) \leq c\right\}
$$

is compact in $C(\bar{D})$ and $W^{1, p}(D), 1 \leq p<\infty$.

(ii) Given $\gamma>0$, the results of part (i) remain true with $P_{D}(\Omega) \leq c$ in place of $P_{\gamma}(\Gamma) \leq c$.

\section{References}

[1] D. Bucur and J.-P. Zolésio, Free boundary problems and density perimeter, J. Differential Equations 126 (1996), 224-243.

[2] M.C. Delfour and J.-P. Zolésio, Shapes and Geometries: Analysis, Differential Calculus and Optimization, SIAM series on Advances in Design and Control, Society for Industrial and Applied Mathematics, Philadelphia, USA 2001.

[3] M.C. Delfour and J.-P. Zolésio, The new family of cracked sets and the image segmentation problem revisited, CRM Report, May 2003, Université de Montréal, accepted in Communications in Information and Systems.

[4] M.C. Delfour, N. Doyon, and J.-P. Zolésio, Extension of the uniform cusp property in shape optimization, in "Control of Partial Differential Equations", G. Leugering, O. Imanuvilov, R. Triggiani, and B. Zhang, eds. Lectures Notes in Pure and Applied Mathematics, Marcel Dekker, May 2003, accepted.

[5] M.C. Delfour, N. Doyon, and J.-P. Zolésio, The uniform fat segment and cusp properties in shape optimization, in "Control and Boundary analysis", J. Cagnol and J.-P. Zolésio (Eds.), pp. 85-96, Marcel Dekker 2004.

[6] E. Giusti, Minimal surfaces and functions of bounded variation, Birkhäuser, Boston, Basel, Stuttgart, 1984. 